\title{
A Brief History of Generative Models for Power Law and Lognormal Distributions
}

\author{
Michael Mitzenmacher
}

Abstract. Recently, I became interested in a current debate over whether file size distributions are best modelled by a power law distribution or a lognormal distribution. In trying to learn enough about these distributions to settle the question, I found a rich and long history, spanning many fields. Indeed, several recently proposed models from the computer science community have antecedents in work from decades ago. Here, I briefly survey some of this history, focusing on underlying generative models that lead to these distributions. One finding is that lognormal and power law distributions connect quite naturally, and hence, it is not surprising that lognormal distributions have arisen as a possible alternative to power law distributions across many fields.

\section{Introduction}

Power law distributions (also often referred to as heavy-tail distributions, Pareto distributions, Zipfian distributions, etc.) are now pervasive in computer science; See, for example, [Broder et al. 00, Crovella and Bestavros 97, Faloutsos et al. 99]. Numerous other examples can be found in the extensive bibliography of this paper.

This paper was specifically motivated by a recent paper by Downey [Downey 01] challenging the now conventional wisdom that file sizes are governed by a

(C) A K Peters, Ltd.

I542-795I/04 $\$ 0.50$ per page 
power law distribution. The argument was substantiated both by collected data and by the development of an underlying generative model which suggested that file sizes were better modeled by a lognormal distribution. ${ }^{1}$ In my attempts to learn more about this question, I was drawn to the history of lognormal and power law distributions. As part of this process, I delved into past and present literature, and came across some interesting facts that appear not to be well known in the computer science community. This paper presents what I have found, focusing specifically on the models of processes that generate these distributions.

Perhaps the most interesting discovery is that much of what we in the computer science community have begun to understand and utilize about power law and lognormal distributions has long been known in other fields, such as economics and biology. For example, models of a dynamically growing Web graph that result in a power law distribution for in- and outdegrees have become the focus of a great deal of recent study. In fact, as I describe below, very similar models date back to at least the 1950s, and arguably back to the 1920s.

A second discovery is the argument over whether a lognormal or power law distribution is a better fit for some empirically observed distribution has been repeated across many fields over many years. For example, the question of whether income distribution follows a lognormal or power law distribution also dates back to at least the 1950s. The issue arises for other financial models, as detailed in [Mandelbrot 97]. Similar issues continue to arise in biology [Jain and Ramakumar 99], chemistry [Nakajima and Higurashi 98], ecology [Allen et al. 01, Sole et al. 00], astronomy [Wheatland and Sturrock 96], and information theory [Li 96, Perline 96]. These cases serve as a reminder that the problems we face as computer scientists are not necessarily new, and we should look to other sciences both for tools and understanding.

A third discovery from examining previous work is that power law and lognormal distributions are intrinsically connected. Very similar basic generative models can lead to either power law or lognormal distributions, depending on seemingly trivial variations. There is, therefore, a reason why this argument as to whether power law or lognormal distributions are more accurate has arisen and repeated itself across a variety of fields.

The purpose of this paper is to explain some of the basic generative models that lead to power law and lognormal distributions, and specifically, to cover how small variations in the underlying model can change the result from one to the other. A second purpose is to provide along the way (incomplete) pointers to some of the recent and historically relevant scientific literature.

${ }^{1}$ I elaborate on this specific model in another paper [Mitzenmacher 02]. 
This survey is intended to be accessible to a general audience; that is, it is intended for computer science theorists, computer scientists who are not theorists, and hopefully also people outside of computer science. Therefore, while mathematical arguments and some probability will be used, the aim is for the mathematics to be intuitive, clean, and comprehensible rather than rigorous and technical. In some cases, details may be suppressed for readability; interested readers are referred to the original papers.

\section{The Distributions: Basic Definitions and Properties}

We begin by reviewing basic facts about power law and lognormal distributions.

For our purposes, a nonnegative random variable $X$ is said to have a power law distribution if

$$
\operatorname{Pr}[X \geq x] \sim c x^{-\alpha}
$$

for constants $c>0$ and $\alpha>0$. Here, $f(x) \sim g(x)$ represents that the limit of the ratios goes to 1 as $x$ grows large. Roughly speaking, in a power law distribution asymptotically the tails fall according to the power $\alpha$. Such a distribution leads to much heavier tails than other common models, such as exponential distributions. One specific commonly used power law distribution is the Pareto distribution, which satisfies

$$
\operatorname{Pr}[X \geq x]=\left(\frac{x}{k}\right)^{-\alpha}
$$

for some $\alpha>0$ and $k>0$. The Pareto distribution requires $X \geq k$. The density function for the Pareto distribution is $f(x)=\alpha k^{\alpha} x^{-\alpha-1}$. For a power law distribution, usually $\alpha$ falls in the range $0<\alpha \leq 2$, in which case, $X$ has infinite variance. If $\alpha \leq 1$, then $X$ also has infinite mean.

If $X$ has a power law distribution, then in a log-log plot of $\operatorname{Pr}[X \geq x]$, also known as the complementary cumulative distribution function, asymptotically the behavior will be a straight line. This provides a simple empirical test for whether a random variable has a power law given an appropriate sample. For the specific case of a Pareto distribution, the behavior is exactly linear, as

$$
\ln (\operatorname{Pr}[X \geq x])=-\alpha(\ln x-\ln k) .
$$

Similarly, on a log-log plot, the density function for the Pareto distribution is also a straight line:

$$
\ln f(x)=(-\alpha-1) \ln x+\alpha \ln k+\ln \alpha .
$$

A random variable $X$ has a lognormal distribution if the random variable $Y=\ln X$ has a normal (i.e., Gaussian) distribution. Recall that the normal 
distribution $Y$ is given by the density function

$$
f(y)=\frac{1}{\sqrt{2 \pi} \sigma} \mathrm{e}^{-(y-\mu)^{2} / 2 \sigma^{2}}
$$

where $\mu$ is the mean, $\sigma$ is the standard deviation ( $\sigma^{2}$ is the variance), and the range is $-\infty<y<\infty$. The density function for a lognormal distribution therefore satisfies

$$
f(x)=\frac{1}{\sqrt{2 \pi} \sigma x} \mathrm{e}^{-(\ln x-\mu)^{2} / 2 \sigma^{2}} .
$$

Note that the change of variables introduces an additional $1 / x$ term outside of the exponential term. The corresponding complementary cumulative distribution function for a lognormal distribution is given by

$$
\operatorname{Pr}[X \geq x]=\int_{z=x}^{\infty} \frac{1}{\sqrt{2 \pi} \sigma z} \mathrm{e}^{-(\ln z-\mu)^{2} / 2 \sigma^{2}} d z .
$$

We will say that $X$ has parameters $\mu$ and $\sigma^{2}$ when the associated normal distribution $Y$ has mean $\mu$ and variance $\sigma^{2}$, where the meaning is clear. The lognormal distribution is skewed, with mean $\mathrm{e}^{\mu+\frac{1}{2} \sigma^{2}}$, median $\mathrm{e}^{\mu}$, and mode $\mathrm{e}^{\mu-\sigma^{2}}$. A lognormal distribution has finite mean and variance, in contrast to the power law distribution under natural parameters.

Despite its finite moments, the lognormal distribution is extremely similar in shape to power law distributions, in the following sense: If $X$ has a lognormal distribution, then in a log-log plot of the complementary cumulative distribution function or the density function, the behavior will appear to be nearly a straight line for a large portion of the body of the distribution. Indeed, if the variance of the corresponding normal distribution is large, the distribution may appear linear on a log-log plot for several orders of magnitude.

To see this, let us look at the logarithm of the density function, which is easier to work with than the complementary cumulative distribution function (although the same idea holds). We have

$$
\begin{aligned}
\ln f(x) & =-\ln x-\ln \sqrt{2 \pi} \sigma-\frac{(\ln x-\mu)^{2}}{2 \sigma^{2}} \\
& =-\frac{(\ln x)^{2}}{2 \sigma^{2}}+\left(\frac{\mu}{\sigma^{2}}-1\right) \ln x-\ln \sqrt{2 \pi} \sigma-\frac{\mu^{2}}{2 \sigma^{2}} .
\end{aligned}
$$

If $\sigma$ is sufficiently large, then the quadratic term of equation (2.2) will be small for a large range of $x$ values, and hence, the logarithm of the density function will appear almost linear for a large range of values.

Finally, recall that normal distributions have the property that the sum of two independent normal random variables $Y_{1}$ and $Y_{2}$ with means $\mu_{1}$ and $\mu_{2}$ and 
variances $\sigma_{1}^{2}$ and $\sigma_{2}^{2}$, respectively, is a normal random variable with mean $\mu_{1}+\mu_{2}$ and variance $\sigma_{1}^{2}+\sigma_{2}^{2}$. It follows that the product of two lognormally distributed random variables also has a lognormal distribution.

\section{Power Laws via Preferential Attachment}

We now move from mathematical definitions and properties to generative models. For the power law distribution, we begin by considering the World Wide Web. The World Wide Web can naturally be thought of as a graph, with pages corresponding to vertices and hyperlinks corresponding to directed edges. Empirical work has shown that indegrees and outdegrees of vertices in this graph obey power law distributions. There has subsequently been a great deal of theoretical work on designing random graph models that yield Web-like graphs [Barabási et al. 99, Broder et al. 00, Cooper and Frieze 01, Drinea et al. 00, Kleinberg et al. 99, Krapivsky and Redner 01, Kumar et al. 99a, Kumar et al 00]. An important criterion for an appropriate random graph model is that it yields power law distributions for the indegrees and outdegrees.

Most models are variations of the following theme. Let us start with a single page, with a link to itself. At each time step, a new page appears, with outdegree 1. With probability $\alpha<1$, the link for the new page points to a page chosen uniformly at random. With probability $1-\alpha$, the new page points to page chosen proportionally to the indegree of the page. This model exemplifies what is often called preferential attachment; new objects tend to attach to popular objects. In the case of the Web graph, new links tend to go to pages that already have links.

A simple, if slightly nonrigorous, argument for the above model goes as follows [Barabási et al. 99, Drinea et al. 00, Krapivsky and Redner 01, Kumar et al 00]. Let $X_{j}(t)$ (or just $X_{j}$ where the meaning is clear) be the number of pages with indegree $j$ when there are $t$ pages in the system. Then, for $j \geq 1$, the probability that $X_{j}$ increases is just

$$
\alpha X_{j-1} / t+(1-\alpha)(j-1) X_{j-1} / t
$$

the first term is the probability a new link is chosen at random and chooses a page with indegree $j-1$, and the second term is the probability that a new link is chosen proportionally to the indegrees and chooses a page with indegree $j-1$. Similarly, the probability that $X_{j}$ decreases is

$$
\alpha X_{j} / t+(1-\alpha) j X_{j} / t .
$$


Hence, for $j \geq 1$, the growth of $X_{j}$ is roughly given by

$$
\frac{d X_{j}}{d t}=\frac{\alpha\left(X_{j-1}-X_{j}\right)+(1-\alpha)\left((j-1) X_{j-1}-j X_{j}\right)}{t} .
$$

Some mathematical purists may object to utilizing a continuous differential equation to describe what is clearly a discrete process. This intuitively appealing approach can be justified more formally using martingales [Kumar et al 00] and in particular, the theoretical frameworks of Kurtz and Wormald [Drinea et al. 00, Kurtz 81, Wormald 95].

The case of $X_{0}$ must be treated specially, since each new page introduces a vertex of indegree 0 .

$$
\frac{d X_{0}}{d t}=1-\frac{\alpha X_{0}}{t} .
$$

Suppose in the steady state limit that $X_{j}(t)=c_{j} \cdot t$; that is, pages of indegree $j$ constitute a fraction $c_{j}$ of the total pages. Then we can successively solve for the $c_{j}$. For example,

$$
\frac{d X_{0}}{d t}=c_{0}=1-\frac{\alpha X_{0}}{t}=1-\alpha c_{0},
$$

from which we find $c_{0}=\frac{1}{1+\alpha}$. More generally, using the equation for $d X_{j} / d t$, we find that for $j \geq 1$,

$$
c_{j}(1+\alpha+j(1-\alpha))=c_{j-1}(\alpha+(j-1)(1-\alpha)) .
$$

This recurrence can be used to determine the $c_{j}$ exactly. Focusing on the asymptotics, we find that for large $j$

$$
\frac{c_{j}}{c_{j-1}}=1-\frac{2-\alpha}{1+\alpha+j(1-\alpha)} \sim 1-\left(\frac{2-\alpha}{1-\alpha}\right)\left(\frac{1}{j}\right) .
$$

Asymptotically, for the above to hold, we have $c_{j} \sim c j^{-\frac{2-\alpha}{1-\alpha}}$ for some constant $c$, giving a power law. To see this, note that $c_{j} \sim c j^{-\frac{2-\alpha}{1-\alpha}}$ implies

$$
\frac{c_{j}}{c_{j-1}} \sim\left(\frac{j-1}{j}\right)^{\frac{2-\alpha}{1-\alpha}} \sim 1-\left(\frac{2-\alpha}{1-\alpha}\right)\left(\frac{1}{j}\right) .
$$

Strictly speaking, to show it is a power law, we should consider $c_{k}^{*}=\sum_{j \geq k} c_{j}$, since we desire the behavior of the tail of the distribution. However, we have

$$
c_{k}^{*} \sim \sum_{j \geq k} c j^{-\frac{2-\alpha}{1-\alpha}} \sim \int_{j=k}^{\infty} c j^{-\frac{2-\alpha}{1-\alpha}} d j \sim c^{\prime} k^{-\frac{1}{1-\alpha}}
$$


for some constant $c^{\prime}$. More generally, if the fraction of items with weight $j$ falls roughly proportionally to $j^{-\alpha}$, the fraction of items with weight greater than or equal to $j$ falls roughly proportionally $j^{1-\alpha}$, a fact we make use of throughout.

Although the above argument was described in terms of degree on the Web graph, this type of argument is clearly very general and applies to any sort of preferential attachment. In fact, the first similar argument dates back to at least 1925. It was introduced by Yule [Yule 25] to explain the distribution of species among genera of plants, which had been shown empirically by Willis to satisfy a power law distribution. While the mathematical treatment from 1925 is different than modern versions, the outline of the general argument is remarkably similar. Mutations cause new species to develop within genera, and more rarely mutations lead to entirely new genera. Mutations within a genus are more likely to occur in a genus with more species, leading to the preferential attachment.

A clearer and more general development of how preferential attachment leads to a power law was given by Simon [Simon 55] in 1955. Again, although Simon was not interested in developing a model for the Web, he lists five applications of this type of model in his introduction: distributions of word frequencies in documents, distributions of numbers of papers published by scientists, distribution of cities by population, distribution of incomes, and distribution of species among genera. Simon was aware of Yule's previous work, and suggests his work is a generalization. Simon's argument, except for notation and the scaling of variables, is painfully similar to the outline above.

As one might expect from Simon's list of applications, power laws had been observed in a variety of fields for some time; Simon was attempting to give a mathematical argument explaining these observations. The earliest apparent reference is to the work by Pareto [Pareto 1896] in 1897, who introduced the Pareto distribution to describe income distribution. The first known attribution of the power law distribution of word frequencies appears to be due to Estoup in 1916 [Estoup 1916], although generally the idea (and its elucidation) are attributed to Zipf [Zipf 32, Zipf 35, Zipf 39]. Similarly, Zipf is often credited with noting that city sizes appear to match a power law, although this idea can be traced back further to 1913 and Auerbach [Auerbach 1913]. Lotka (circa 1926) found in examining the number of articles produced by chemists that the distribution followed a power law [Lotka 26]; indeed, power laws of various forms appear in many places in informetrics [Bookstein 90].

Although we now associate the argument above with the Web graph, even before the Web graph became popular, more formal developments of the argument above had been developed as part of the study of random trees. Specifically, consider the following recursive tree structure. Begin with a root node. At each step, a new node is added; its parent is chosen from the current vertices with 
probability proportional to one plus the number of children of the node. This is just another example of preferential attachment; indeed, it is essentially equivalent to the simple Web graph model described above with the probability $\alpha$ of choosing a random node equal to $1 / 2$. That the degree distribution of such graphs obey a power law (in expectation) was proven in 1993 in works by Mahmoud, Smythe, and Szymański [Mahmound et al. 93]. See also the related [Lu and Feng 98, Szymański 87, Pittel 94, Smythe and Mahmound 95].

Of course, in recognizing the relationship between the recent work on Web graph models and this previous work, it would be remiss to not point out that modern developments have led to many new insights. Perhaps most important is the development of a connection between Simon's model, which appears amenable only to limiting analysis based on differential equations, and purely combinatorial models based on random graphs [B. Bollobás et al. 01, Mahmound et al. 93, Smythe and Mahmound 95]. Such a connection is important for further rigorous analysis of these structures. Also, current versions of Simon's arguments based on martingales provide a much more rigorous foundation [B. Bollobás et al. 01, Cooper and Frieze 01, Kumar et al 00, Lu and Feng 98]. More recent work has focused on greater understanding of the structure of graphs that arise from these kinds of preferential attachment model. It has been shown that in the Web graph model described above, where new pages copy existing links, the graphs have community substructures [Kumar et al 00], a property not found in random graphs, but amply found in the actual Web [Gibson et al. 98, Kumar et al. 99b]. The diameter of these random Web graphs have also been the subject of recent study [Albert et al. 99, Bollobás and Riordan to appear]. Still, it is important to note how much was already known about the power law phenomenon in various fields well before the modern effort to understand power laws on the Web, and how much computer scientists had to reinvent.

\section{Power Laws via Optimization}

Mandelbrot had developed other arguments for deriving power law distributions based on information theoretic considerations somewhat earlier than Simon [Mandelbrot 53]. His argument is very similar in spirit to other recent optimization-based arguments for heavy tailed distributions [Carlson and Doyle 99, Fabrikant et al. 02, Zhu et al. 01].

We sketch Mandelbrot's framework, which demonstrates a power law in the rank-frequency distribution of words. That is, the frequency $p_{j}$ of the $j$ th mostused word, given as a fraction of the time that word appears, follows a power law in $j$, so $p_{j} \sim c j^{-\alpha}$. This is a slightly different flavor than the type power law 
than we considered previously; Simon's model considers the fraction of words that appear $j$ times. But, of course, the two are related. We clarify this by following an argument of Bookstein [Bookstein 90].

Suppose we have a text where the number of words $q_{k}$ that appear $k$ times is given by $q_{k}=Q k^{-\alpha}$ for $\alpha>1$. Further, suppose for convenience we have one most frequent word that appears $k_{m}$ times, so that we may write $q_{k}=\left(k / k_{m}\right)^{-\alpha}$. The number of words that appear $k$ or more times is then approximately

$$
\int_{k}^{k_{m}}\left(\frac{x}{k_{m}}\right)^{-\alpha} d x
$$

and hence, the rank $j$ of a word that appears $k$ times is approximately

$$
j=\frac{j_{m}}{\alpha-1}\left[\left(\frac{j_{m}}{k}\right)^{\alpha-1}-1\right] .
$$

Now solving for $k$ in terms of $j$, we find that the $j$ th most-used word appears approximately

$$
k=j_{m}\left[\frac{(\alpha-1) j}{j_{m}}+1\right]^{-1 /(\alpha-1)}
$$

times, yielding a power law for the frequency $p_{j}$ as a function of $j$.

We now begin Mandelbrot's argument. Consider some language consisting of $n$ words. The cost of using the $j$ th word of the language in a transmission is $C_{j}$. For example, if we think of English text, the cost of a word might be thought of as the number of letters plus the additional cost of a space. Hence, a natural cost function has $C_{j} \sim \log _{d} j$ for some alphabet size $d$. Suppose that we wish to design the language to optimize the average amount of information per unit transmission cost. Here, we take the average amount of information to be the entropy. We think of each word in our transmission as being selected randomly, and the probability that a word in the transmission is the $j$ th word of the language is $p_{j}$. Then, the average information per word is the entropy $H=-\sum_{j=1}^{n} p_{j} \log _{2} p_{j}$, and the average cost per word is $C=\sum_{j=1}^{n} p_{j} C_{j}$. The question is how would the $p_{j}$ be chosen to minimize $A=C / H$. Taking derivatives, we find

$$
\frac{d A}{d p_{j}}=\frac{C_{j} H+C \log _{2}\left(\mathrm{e} p_{j}\right)}{H^{2}} .
$$

Hence, all the derivatives are 0 (and $A$ is, in fact, minimized) when $p_{j}=$ $2^{-H C_{j} / C} /$ e. Using $C_{j} \sim \log _{d} j$, we obtain a power law for the $p_{j}{ }^{2}$ Mandel-

\footnotetext{
${ }^{2}$ The eagle-eyed reader might note that technically the result above does not quite match a power law as we have defined it; just because $C_{j} \sim \log _{d} j$ does not strictly give us $p_{j} \sim j^{-\alpha}$. In this case, this is a minor point; really $C_{j}$ is within an additive constant of $\log _{d} j$, and we therefore find that $p_{j}$ is within a constant multiplicative factor of a power law. We ignore this distinction henceforth.
} 
brot argues that a variation of this model matches empirical results for English quite well.

Carlson and Doyle suggest a similar framework for analyzing file sizes and forest files [Carlson and Doyle 99]. Fabrikant, Koutsoupias, and Papadimitriou introduce combinatorial models for the Internet graph (which should not be confused with the Web graph; the Internet graph consists of the servers and links between them as opposed to Web pages) and file sizes based on local optimization that also yield power laws [Fabrikant et al. 02].

As an aside, I found when reviewing the literature that Mandelbrot strongly argued against Simon's alternative assumptions and derivations based on preferential attachment when his article came out. This led to what is in retrospect an amusing but apparently at the time quite heated exchange between Simon and Mandelbrot in the journal Information and Control [Mandelbrot 59, Simon 60, Mandelbrot 61a, Simon 61a, Mandelbrot 61b, Simon 61b]. ${ }^{3}$

It is worth noting that economists appear to have given the nod to Simon and the preferential attachment model. Indeed, a recent popular economics text by Krugman [Krugman 96] offers a derivation of the power law similar to Simon's argument. ${ }^{4}$ A more formal treatment is given by Gabaix [Gabaix 99].

\section{Multiplicative Processes}

Lognormal distributions are generated by processes that follow what the economist Gibrat called the law of proportionate effect [Gibrat 30, Gibrat 31]. We use the term multiplicative process to describe the underlying model. In biology, such processes are used to described the growth of an organism. Suppose we start with an organism of size $X_{0}$. At each step $j$, the organism may grow or shrink, according to a random variable $F_{j}$, so that

$$
X_{j}=F_{j} X_{j-1} .
$$

\footnotetext{
${ }^{3} \mathrm{~A}$ few excerpts from the exchange are worth citing to demonstrate the disagreement. The abstract of Mandelbrot's initial note begins, "This note is a discussion of H. A. Simon's model (1955) concerning the class of frequency distributions generally associated with the name of G. K. Zipf. The main purpose is to show that Simon's model is analytically circular in the case of the linguistic laws of Estouf-Zipf and Willis-Yule." [Mandelbrot 59] The abstract of Simon's rebuttal begins, "This note takes issue with a recent criticism by Dr. B. Mandelbrot of a certain stochastic model to explain word-frequency data. Dr. Mandelbrot's principal empirical and mathematical objections to the model are shown to be unfounded." The subsequent abstracts are similar in spirit.

${ }^{4} \mathrm{As}$ an interesting example of the pervasiveness of power-law behavior, one review of Krugman's book, written by an urban geographer, accuses the author of excessive hubris for not noting the significant contributions made by urban geographers with regard to Simon's model [Berry 99].
} 
The idea is that the random growth of an organism is expressed as a percentage of its current weight, and is independent of its current actual size. If the $F_{k}, 1 \leq$ $k \leq j$, are all governed by independent lognormal distributions, then so is each $F_{j}$, inductively, since the product of lognormal distributions is again lognormal.

More generally, approximately lognormal distributions may be obtained even if the $F_{j}$ are not themselves lognormal. Specifically, consider

$$
\ln X_{j}=\ln X_{0}+\sum_{k=1}^{j} \ln F_{k}
$$

Assuming the random variables $\ln F_{k}$ satisfy appropriate conditions, the Central Limit Theorem says that $\sum_{k=1}^{j} \ln F_{k}$ converges to a normal distribution, and hence, for sufficiently large $j, X_{j}$ is well approximated by a lognormal distribution. In particular, if the $\ln F_{k}$ are independent and identically distributed variables with finite mean and variance, then asymptotically $X_{j}$ will approach a lognormal distribution.

Multiplicative processes are used in biology and ecology to describe the growth of organisms or the population of a species. In economics, perhaps the most wellknown use of the lognormal distribution derives from the Black-Scholes option pricing model [Black and Scholes 73], which is a specific application of Ito's lemma (see, e.g., [Hull 97, Ito 51]). In a simplified version of this setting [Cox et al. 79, Hull 97], the price of a security moves in discrete time steps, and the price $X_{j}$ changes according to $X_{j}=F_{j} X_{j-1}$, where $F_{j}$ is lognormally distributed. Using this model, Black and Scholes demonstrate how to use options to guarantee a risk-free return equivalent to the prevailing interest rate in a perfect market. Other applications in, for example, geology and atmospheric chemistry are given in [Crow and Shimizu 88]. More recently, as described below, Adamic and Huberman suggest that multiplicative processes may describe the growth of sites on the Web as well as the growth of user traffic on web sites [Huberman and Adamic 99, Huberman and Adamic 00]. Lognormal distributions have also been suggested for file sizes [Barford et al. 99, Barford and Crovella 98, Downey 01].

The connection between multiplicative processes and the lognormal distribution can be traced back to Gibrat around 1930 [Gibrat 30, Gibrat 31], although Kapteyn described, in other terms, an equivalent process in 1903 [Kapteyn 1903], and McAlister described the lognormal distribution around 1879 [McAlister 1879]. Aitchison and Brown suggest that the lognormal distribution may be a better fit for income distribution than a power law distribution, representing perhaps the first time the question of whether a power law distribution or a lognormal distribution gives a better fit was fully developed [Aitchison and Brown 
54, Aitchison and Brown 57]. It is interesting that when examining income distribution data, Aitchison and Brown observe that for lower incomes a lognormal distribution appears a better fit, while for higher incomes a power law distribution appears better; this is echoed in later work by Montroll and Schlesinger [Montroll and Schlesinger 82, Montroll and Schlesinger 83], who offer a possible mathematical justification discussed below. Similar observations have been given for file sizes [Barford et al. 99, Barford and Crovella 98].

\section{I. Multiplicative Models and Power Law Distributions}

Although the multiplicative model is used to generate lognormal or approximately distributions, only a small change from the lognormal generative process yields a generative process with a power law distribution. To provide a concrete example, we consider the interesting history of work on income distributions.

Recall that Pareto introduced the Pareto distribution in order to explain income distribution at the tail end of the nineteenth century. Champernowne [Champernowne 53], in a work slightly predating Simon (and acknowledged by Simon, who suggested his work generalized and extended Champernowne), offered an explanation for this behavior. Suppose that we break income into discrete ranges in the following manner. We assume there is some minimum income $m$. For the first range, we take incomes between $m$ and $\gamma m$, for some $\gamma>1$; for the second range, we take incomes between $\gamma m$ and $\gamma^{2} m$. We therefore say that a person is in class $j$ for $j \geq 1$ if their income is between $m \gamma^{j-1}$ and $m \gamma^{j}$. Champernowne assumes that over each time step, the probability of an individual moving from class $i$ to class $j$, which we denote by $p_{i j}$, depends only on the value of $j-i$. He then considers the equilibrium distribution of people among classes. Under this assumption, Pareto distributions can be obtained.

Let us examine a specific case, where $\gamma=2, p_{i j}=2 / 3$ if $j=i-1$, and $p_{i j}=1 / 3$ if $j=i+1$. Of course, the case $i=1$ is a special case; in this case, $p_{11}=2 / 3$. In this example, outside of class 1 , the expected change in income over any step is 0 . It is also easy to check that, in this case, the equilibrium probability of being in class $k$ is just $1 / 2^{k}$, and hence, the probability of being in class greater than or equal to $k$ is $1 / 2^{k-1}$. Hence the probability that a person's income $X$ is larger than $2^{k-1} m$ in equilibrium is given by

$$
\operatorname{Pr}\left[X \geq 2^{k-1} m\right]=1 / 2^{k-1},
$$

or

$$
\operatorname{Pr}[X \geq x]=m / x
$$

for $x=2^{k-1} m$. This is a power law distribution. 
Note, however, the specific model above looks remarkably like a multiplicative model. Moving from one class to another can be thought of as either doubling or halving your income over one time step. That is, if $X_{t}$ is your income after $t$ time steps, then

$$
X_{t}=F_{t} X_{t-1},
$$

where $F_{t}$ is $1 / 2$ with probability $2 / 3$ and 2 with probability $1 / 3$. Again, $E\left[X_{t}\right]=$ $E\left[X_{t-1}\right]$. Our previous discussion therefore suggests that $X_{t}$ should converge to a lognormal distribution for large $t$.

What is the difference between the Champernowne model and the multiplicative model? In the multiplicative model, income can become arbitrarily close to zero through successive decreases; in the Champernowne model, there is a minimum income corresponding to the lowest class below which one cannot fall. This small change allows one model to produce a power law distribution while the other produces a lognormal. As long as there is a bounded minimum that acts as a lower reflective barrier to the multiplicative model, it will yield a power law instead of a lognormal distribution. The theory of this phenomenon is more fully developed in [Gabaix 99, Kesten 73].

\section{Monkeys Typing Randomly}

We return now to Mandelbrot's optimization argument for the power law behavior of word frequency in written language. A potentially serious objection to Mandelbrot's argument was developed by the psychologist Miller [Miller 57], who demonstrated that the power law behavior of word frequency arises even without an underlying optimization problem. This result, explained below, should perhaps serve as warning: Just because one finds a compelling mechanism to explain a power law does not mean that there are not other, perhaps simpler, explanations.

Miller suggests the following experiment. A monkey types randomly on a keyboard with $n$ characters and a space bar. A space is hit with probability $q$; all other characters are hit with equal probability $(1-q) / n$. A space is used to separate words. We consider the frequency distribution of words.

It is clear that, as the monkey types, each word with $c$ (nonspace) characters occurs with probability

$$
q_{c}=\left(\frac{1-q}{n}\right)^{c} q,
$$

and there are $n^{c}$ words of length $c$. (We allow the empty word of length 0 for convenience.) The words of longer length are less likely and hence, occur lower in 
the rank order of word frequency. In particular, the word with frequency ranks $1+\left(n^{j}-1\right) /(n-1)$ to $\left(n^{j+1}-1\right) /(n-1)$ have $j$ letters. Hence, the word with frequency rank $r_{j}=n^{j}$ occurs with probability

$$
q_{j}=q\left(\frac{1-q}{n}\right)^{\log _{n} r_{j}}=q\left(r_{j}\right)^{\log _{n}(1-q)-1},
$$

and the power law behavior is apparent. Hence, the power law associated with word frequency requires neither preferential attachment nor optimization; monkeys typing randomly would produce it.

Bell, Cleary, and Witten observe empirically that when the probabilities of each letter are not equal, a smoother match to the power law develops [Bell et al. 90]. I am currently unaware of a proof similar to the one above demonstrating that power law behavior occurs when the probabilities for each of the letters are arbitrary. Indeed, to confuse the issue, one paper on the subject claims that if the letter frequencies are not equal, a lognormal distribution occurs [Perline 96] (see also [Gong et al. 01], where this claim is repeated). It is worth examining this argument more carefully, since it demonstrates the confusion that can arise in trying to distinguish models that generate power law and lognormal distributions.

Perline notes that in the experiment with monkeys typing randomly, if we consider words only of some fixed length $m$, for $m$ sufficiently large their frequencyrank distribution will approximate a lognormal distribution, following the paradigm of multiplicative processes. To see this, let the probabilities for our $n$ characters be $p_{1}, p_{2}, \ldots, p_{n}$. Consider the generation a random $m$-letter word. Let $X_{i}$ take on the value $p_{j}$ if the $i$ th letter is $j$. Then $Y_{m}=X_{1} X_{2} \ldots X_{m}$ is a random variable whose value corresponds to the probability that a word chosen uniformly at random from all $m$-letter words appears as the monkeys type. We have that $\ln Y_{m}=\sum_{k=1}^{m} \ln X_{i}$; since the $X_{i}$ are independent and identically distributed, $\ln Y_{m}$ converges to a normal distribution by the Central Limit Theorem, and hence, $Y_{m}$ converges to a lognormal distribution. Notice that this holds true even if all letter frequencies are equal, although in this case, the resulting distribution is trivial.

Perline then argues that if we consider all words of length up to $m$, we still obtain asymptotic convergence to a lognormal distribution. This follows from a generalization of the Central Limit Theorem due to Anscombe. Intuitively, this is because most words have length close to $m$, so the words with small length are just noise in the distribution. This result requires that some two letters have different probabilities of being hit.

From this, it might be tempting to conclude that the distribution, if the word length is unrestricted, is also lognormal when letters do not all have the same 
probabilities. However, this does not follow. The problem is that for each value of $m$ we obtain a slightly different lognormal distribution. Hence, it is not necessarily true that in the limit as $m$ increases we are getting closer and closer to some final lognormal distribution. Rather, we have a sequence of lognormal distributions that is converging to some distribution. To justify that the result need not be lognormal, I present an amusing example of my own devising.

Consider an alphabet with two letters: "a" occurs with probability $q$, "b" occurs with probability $q^{2}$, and a space occurs with probability $1-q-q^{2}$. The value $q$ must be chosen so that $1-q-q^{2}>0$. In this case, every valid word the monkey can type occurs with probability $q^{j}\left(1-q-q^{2}\right)$ for some integer $j$. Let us say a word has pseudorank $j$ if it occurs with probability $q^{j}\left(1-q-q^{2}\right)$. There is 1 word with pseudorank 0 (the empty word), 1 with pseudorank 1 ("a"), 2 with pseudorank 2 ("aa" and "b"), and so on. A simple induction yields that the number of words with pseudorank $k$ is in fact the $(k+1)$ st Fibonacci number $F_{k+1}$ (where here we start with $F_{0}=0$ and $F_{1}=1$ ). This follows obviously from the fact that to obtain the words with pseudorank $k$ we append an "a" to a word with pseudorank $k-1$, or a "b" to a word with pseudorank $k-2$.

Recall that $F_{k} \approx \Phi^{k} / \sqrt{5}$ for large $k$, where $\Phi=(1+\sqrt{5}) / 2$. Also $\sum_{i=1}^{k} F_{k}=$ $F_{k+2}-1$. Now the argument is entirely similar to the case where all items have the same probability. If we ask for the frequency of the $r_{j}=F_{j}$ th most frequent item, it has pseudorank $j-2$, and hence its frequency is

$$
q^{j-2}\left(1-q-q^{2}\right) \approx q^{\log _{\Phi} \sqrt{5} r_{j}-2}\left(1-q-q^{2}\right)=r_{j}^{\log _{\Phi} q} q^{\log _{\Phi} \sqrt{5}-2}\left(1-q-q^{2}\right),
$$

and again we have power law behavior.

There is nothing special about having two characters for this example; one could easily expand it to include more complex generalized Fibonacci sequences. A suitable generalization appears feasible for any probabilities $p_{1}, p_{2}, \ldots, p_{n}$ associated with the $n$ characters, although a formal proof is beyond the scope of this survey. ${ }^{5}$ Roughly, let $p_{1}$ be the largest of the $p_{i}$, and let $p_{j}=p_{1}^{\gamma_{j}}$ for $j \geq 1$. Then the number of words with frequency greater than or equal to $p_{1}^{k}$ grows approximately proportionally to $(1 / c)^{k}$, where $c$ is the unique real root between 0 and 1 of $\sum_{j=1}^{n} x^{\gamma_{j}}=1$. This is all we need for the monkeys to produce a power law distribution, following the arguments above.

\footnotetext{
${ }^{5}$ Brian Conrad and I have constructed a formal treatment of this argument, using some nontrivial analytic number theory. This work has been submitted for publication.
} 


\section{Double Pareto Distributions}

Interestingly, there is another variation on the multiplicative generative model that yields power law behavior. Recall that in the multiplicative model, if we begin with value $X_{0}$ and every step yields an independent and identically distributed multiplier from a lognormal distribution $F$, then any resulting distribution $X_{t}$ after $t$ steps is lognormal. Suppose, however, that instead of examining $X_{t}$ for a specific value of $t$, we examine the random variable $X_{T}$ where $T$ itself is a random variable. As an example, when considering income distribution, in seeing the data we may not know how long each person has lived. If different age groups are intermixed, the number of multiplicative steps each person may be thought to have undergone may be thought of as a random variable.

This effect was noticed as early as 1982 by Montroll and Schlesinger [Montroll and Schlesinger 82, Montroll and Schlesinger 83]. They show that a mixture of lognormal distributions based on a geometric distribution would have essentially a lognormal body but a power law distribution in the tail. Huberman and Adamic suggest a pleasantly simple variation of the above result; in the case where the time $T$ is an exponential random variable, and we may think of the number of multiplicative steps as being continuous, the resulting distribution of $X_{T}$ has a power law distribution [Huberman and Adamic 99, Huberman and Adamic 00]. Huberman and Adamic go on to suggest that this result can explain the power law distribution observed for the number of pages per site. As the Web is growing exponentially, the age of a site can roughly be thought of as distributed like an exponential random variable. If the growth of the number of pages on a Web site follows a multiplicative process, the above result suggests a power law distribution.

In more recent independent work, Reed provides the correct full distribution for the above model, which yields what he calls a double Pareto distribution [Reed 00]. Specifically, the resulting distribution has one Pareto tail distribution for small values (below some point) and another Pareto tail distribution for large values (above the same point). ${ }^{6}$

For example, consider for simplicity the case where if we stop a process at time $t$ the result is a lognormal random variable with mean 0 and variance $t$. Then, if we stop the process at an exponentially distributed time with mean $1 / \lambda$, the density function of the result is

$$
f(x)=\int_{t=0}^{\infty} \lambda \mathrm{e}^{-\lambda t} \frac{1}{\sqrt{2 \pi t} x} \mathrm{e}^{-(\ln x)^{2} / 2 t} d t .
$$

\footnotetext{
${ }^{6}$ For completeness, we note that Huberman and Adamic concentrate only on the tail of the density function, and correctly determine the power law behavior. However, they miss the two-sided nature of the distribution. Reed gives the complete correct form, as we do below.
} 
Using the substitution $t=u^{2}$ gives

$$
f(x)=\frac{2 \lambda}{\sqrt{2 \pi} x} \int_{u=0}^{\infty} \mathrm{e}^{-\lambda u^{2}-(\ln x)^{2} / 2 u^{2}} d u .
$$

An integral table gives us the identity

$$
\int_{z=0}^{\infty} \mathrm{e}^{-a z^{2}-b / z^{2}}=\frac{1}{2} \sqrt{\frac{\pi}{a}} \mathrm{e}^{-2 \sqrt{a b}},
$$

which allows us to solve for the resulting form. Note that in the exponent $\sqrt{2 a b}$ of the identity we have $b=(\ln x)^{2} / 2$. Because of this, there are two different behaviors, depending on whether $x \geq 1$ or $x \leq 1$. For $x \geq 1, f(x)=$ $(\sqrt{\lambda / 2}) x^{-1-\sqrt{2 \lambda}}$, so the result is a power law distribution. For $x \leq 1, f(x)=$ $(\sqrt{\lambda / 2}) x^{-1+\sqrt{2 \lambda}}$.

The double Pareto distribution falls nicely between the lognormal distribution and the Pareto distribution. Like the Pareto distribution, it is a power law distribution. But while the log-log plot of the density of the Pareto distribution is a single straight line, for the double Pareto distribution the log-log plot of the density consists of two straight line segments that meet at a transition point. This is similar to the lognormal distribution, which has a transition point around its median $\mathrm{e}^{\mu}$ due to the quadratic term, as shown in Equation (2.1). Hence, an appropriate double Pareto distribution can closely match the body of a lognormal distribution and the tail of a Pareto distribution. For example, Figure 1 shows the complementary cumulative distribution function for a lognormal and a double Pareto distribution. (These graphs have only been minimally tuned to give a reasonable match.) The plots match quite well with a standard scale for probabilities, as shown on the left. On the log-log scale, however, one can see the difference in the tail behavior. The double Pareto distribution follows a power law; the lognormal distribution has a clear curvature.

Reed also suggests a generalization of the above called a double Pareto-lognormal distribution with similar properties [Reed 01]. The double Pareto-lognormal distribution has more parameters, but might allow closer matches with empirical distributions.

It seems reasonable that in many processes the time an object has lived should be considered a random variable as well, and hence, this model may prove more accurate for many situations. For example, that the double Pareto tail phenomenon could explain why income distributions and file size distributions appear better modeled by a distribution with a lognormal body and a Pareto tail [Aitchison and Brown 54, Barford et al. 99, Barford and Crovella 98, Montroll and Schlesinger 82, Montroll and Schlesinger 83]. Reed presents empirical evidence 

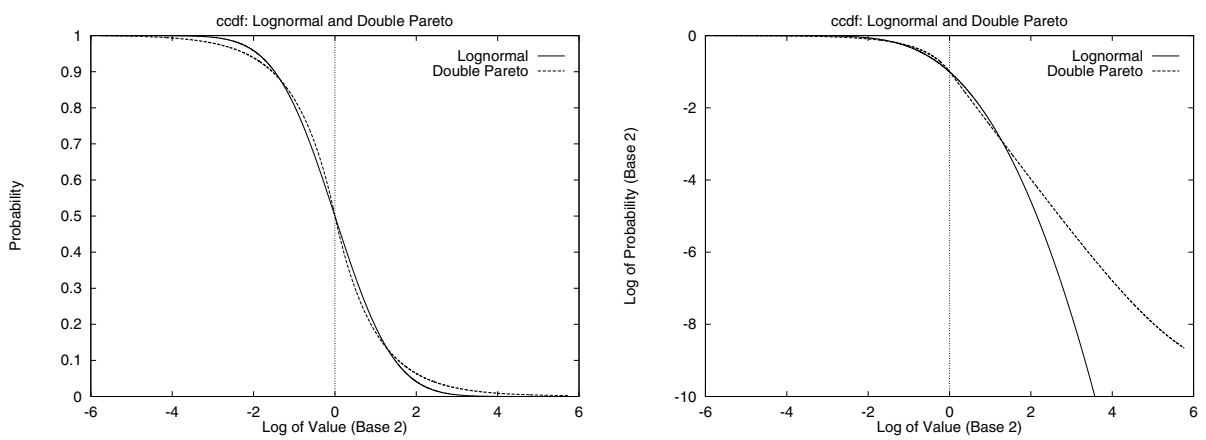

Figure I. Shapes of lognormal and double Pareto distributions.

in favor of using the double Pareto and double-Pareto lognormal distributions for incomes and other applications [Reed 00, Reed 01].

To give an idea of why it might be natural for the time parameter to be (roughly) exponentially distributed, I briefly describe a model that I introduced in [Mitzenmacher 02]. This model combines ideas from the theory of recursive trees, preferential attachment, and the double Pareto framework. Consider a graph process that works as follows: At each step, with probability $\gamma$, a new node is introduced that becomes the root of a new tree. Each new node has an associated size chosen independently and uniformly at random from a distribution $\mathcal{D}_{1}$. With probability $1-\gamma$, an existing node is chosen uniformly at random, and it generates a child. The size of a child is equal to the size of its parent, multiplied by some multiplicative factor chosen by a distribution $\mathcal{D}_{2}$. It is easy to show that the distribution of the depths of the nodes generated in this manner converges to a geometric distribution. Along each branch of the tree, the size of the nodes follows a multiplicative process. If $\mathcal{D}_{1}$ and $\mathcal{D}_{2}$ are lognormal distributions, then the size of a randomly chosen node is a geometric mixture of lognormally distributed random variables, which closely matches the exponential mixture required for a double Pareto distribution. In fact, the tail behaviors are the same. I use this model to explain file size distributions in [Mitzenmacher 02]; [Reed and Hughes 02] analyzes other similar models.

This line of thought also ties back into the discussion of monkeys typing randomly. In the case of unrestricted word lengths and unequal letter probabilities, the word length is geometrically distributed, and the probability of a word of any (large) fixed length is approximately lognormal, with the appropriate mean and variance being proportional to the length of the word. Hence, the underlying distribution of word lengths is a geometric mixture of approximately lognormal 
random variables as in the framework above, and hence, the resulting power law is unsurprising.

\section{Conclusions}

Power law distributions and lognormal distributions are quite natural models and can be generated from simple and intuitive generative processes. Because of this, they have appeared in many areas of science. This example should remind us of the importance of seeking out and recognizing work in other disciplines, even if it lies outside our normal purview. Since computer scientists invented search engines, we really have little excuse. On a personal note, I was astounded at how the Web and search engines have transformed the possibilities for mining previous research; many of the decades-old articles (including the 1925 article by Yule!) cited here are, in fact, available on the Web.

It is not clear that the above discussion settles one way or another whether lognormal or power law distributions are better models for things like file size distributions. Given the close relationship between the two models, it is not clear that a definitive answer is possible; it may be that in seemingly similar situations slightly different assumptions prevail. The fact that power law distributions arise for multiplicative models once the observation time is random or a lower boundary is put into effect, however, may suggest that power laws are more robust models. Indeed, following the work of Reed [Reed 00, Reed 01], we recommend the double Pareto distribution and its variants as worthy of further consideration in the future.

From a more pragmatic point of view, it might be reasonable to use whichever distribution makes it easier to obtain results. This runs the risk of being inaccurate; perhaps in some cases, the fact that power law distributions can have infinite mean and variance are salient features, and therefore substituting a lognormal distribution loses this important characteristic. Also, if one is attempting to predict future behavior based on current data, misrepresenting the tail of the distribution could have severe consequences. For example, large files above a certain size might be rare currently, and hence, both lognormal and power law distibutions based on current data might capture these rare events adequately. As computer systems with more memory proliferate, and even larger files become more frequent, the prediction from two models may vary more substantially. The recent work [Gong et al. 01] argues that for at least some network applications the difference in tails is not important. We believe that formalizing this idea is an important open question. Specifically, it would be useful to know in a more formal sense in what situations the small differences between power laws 
and lognormal distributions manifest themselves in vastly different qualitative behavior, and in what cases a power law distribution can be suitably approximated by lognormal distributions.

For further reading, I strongly recommend the article by Xavier Gabaix [Gabaix 99], which provides both underlying mathematics and an economic perspective and history. Similarly, Mandelbrot provides both history about and his own perspective on lognormal and power law distributions in a recent book [Mandelbrot 97]. Wentian Li has a web page devoted to Zipf's law which is an excellent reference [Li 99]. For lognormal distributions, useful sources include the text by Aitchison and Brown [Aitchison and Brown 57] or the modern compendium edited by Crow and Shimizu [Crow and Shimizu 88].

Acknowledgments. I would like to thank John Byers, Mark Crovella, Allen Downey, Alan Frieze, Jon Kleinberg, and Eli Upfal for suggestions on how to improve this work. This paper is supported in part by an Alfred P. Sloan Research Fellowship and NSF grant CCR-9983832.

\section{References}

[Aiello et al. 00] W. Aiello, F. Chung, and L. Lu. "A Random Graph Model for Massive Graphs." In Proceedings of the 32nd Annual ACM Symposium on Theory of Computing, pp. 171-180. New York: ACM Press, 2000.

[Aitchison and Brown 54] J. Aitchison and J. A. C. Brown. "On Criteria for Descriptions of Income Distribution." Metroeconomica 6 (1954), 88-98.

[Aitchison and Brown 57] J. Aitchison and J. A. C. Brown. The Lognormal Distribution. Cambridge, UK: Cambridge University Press, 1957.

[Allen et al. 01] A. P. Allen, B. Li, and E. L. Charnov. "Population Fluctuations, Power Laws and Mixtures of Lognormal Distributions." Ecology Letters 4 (2001), $1-3$.

[Albert et al. 99] R. Albert, H. Jeong, and A.-L. Barabási. "Diameter of the World Wide Web." Nature 401 (1999), 130-131.

[Auerbach 1913] F. Auerbach. "Das Gesetz der Bevolkerungskonzentration." Petermanns Geographische Mitteilungen LIX (1913), 73-76.

[Barabási et al. 99] A.-L. Barabási, R. Albert, and H. Jeong. "Mean-Field Theory for Scale-Free Random Networks." Physica A 272 (1999), 173-189.

[Barford et al. 99] P. Barford, A. Bestavros, A. Bradley, and M. Crovella. "Changes in Web Client Access Patterns: Characteristics and Caching Implications." World Wide Web 2 (1999), 15-28.

[Barford and Crovella 98] P. Barford and M. Crovella. "Generating Representative Web Workloads for Network and Server Performance Evaluation." In Proceedings of ACM SIGMETRICS, pp. 151-160. New York: ACM Press, 1998. 
[Bell et al. 90] T. C. Bell, J. G. Cleary, and I. H. Witten. Text Compression. Englewood Cliffs, NJ: Prentice-Hall, 1990.

[Berry 99] B. Berry. "Déjà vu, Mr. Krugman." Urban Geography 20 (1999) 1-2.

[Black and Scholes 73] F. Black and M. Scholes. "The Pricing of Options and Corporate Liabilities." Journal of Political Economics 81 (1973), 637-654.

[Bollobás and Riordan to appear] B. Bollobás and O. Riordan. "The Diameter of a Scale-Free Random Graph." To appear.

[B. Bollobás et al. 01] B. Bollobás, O. Riordan, J. Spencer, and G. Tusnády. "The Degree Sequence of a Scale-Free Random Process." Random Structures and Algorithms 18:3 (2001), 279-290.

[Bookstein 90] A. Bookstein. "Informetric Distributions, Part I: Unified Overview." Journal of the American Society for Information Science 41:5 (1990), 368-375.

[Broder et al. 00] A. Broder, R. Kumar, F. Maghoul, P. Raghavan, S. Rajagopalan, R. Stata, A. Tomkins, and J. Wiener. "Graph Structure in the Web: Experiments and Models." In Proc. of the 9th World Wide Web Conference, pp. 309-320. Amsterdam: Elsevier Science, 2000.

[Carlson and Doyle 99] J. M. Carlson and J. Doyle. "Highly Optimized Tolerance: A Mechanism for Power Laws in Designed Systems." Physics Review E 60:2 (1999), 1412-1427.

[Champernowne 53] D. Champernowne. "A Model of Income Distribution." Economic Journal 63 (1953), 318-351.

[Cooper and Frieze 01] C. Cooper and A. Frieze. "On a General Model of Undirected Web Graphs." In Proceedings of the 9th Annual European Symposium on Algorithms, pp. 500-511. Berlin-Heidelberg: Springer-Verlag, 2001.

[Cox et al. 79] J. Cox, S. Ross, and M. Rubinstien. "Option Pricing: A Simplified Approach." Journal of Financial Economics 7 (1979), 229-265.

[Crovella and Bestavros 97] M. Crovella and A. Bestavros. "Self-Similarity in World Wide Web Traffic: Evidence and Possible Causes." IEEE/ACM Transactions on Networking 5:6 (1997), 835-846.

[Crovella et al. 98] M. Crovella, M. S. Taqqu, and A. Bestavros. "Heavy-Tailed Probability Distributions in the World Wide Web." In A Practical Guide to Heavy Tails, edited by R. J. Adler, R. E. Feldman, and M. S. Taqqu, pp. 3-26. London: Chapman and Hall, 1998.

[Crow and Shimizu 88] E. L. Crow and K. Shimizu (editors). Lognormal Distributions: Theory and Applications. New York: Markel Dekker, Inc., 1988.

[Drinea et al. 00] E. Drinea, M. Enachescu, and M. Mitzenmacher. "Variations on Random Graph Models of the Web." Harvard Computer Science Technical Report TR-06-01, 2000.

[Downey 01] A. B. Downey. "The Structural Causes of File Size Distributions." To appear in MASCOTS 2001. Available from World Wide Web (http://rocky.wellesley.edu/downey/filesize/), 2001. 
[Estoup 1916] J. B. Estoup. Gammes Stenographiques. Paris: Institut Stenographique de France, 1916.

[Fabrikant et al. 02] A. Fabrikant, E. Koutsoupias, and C. H. Papadimitriou. "Heuristically Optimized Tradeoffs: A New Paradigm for Power Laws in the Internet." In Proceedings of the 29th International Colloquium on Automata, Languages, and Programming, Lecture Notes in Computer Science 2380, pp. 110-122. BerlinHeidelberg: Springer-Verlag, 2002.

[Faloutsos et al. 99] M. Faloutsos, P. Faloutsos, and C. Faloutsos. "On Power-Law Relationships of the Internet Topology." In Proceedings of the ACM SIGCOMM 1999 Conference, pp. 251-261. New York: ACM Press, 1999.

[Gabaix 99] X. Gabaix. "Zipf's Law for Cities: An Explanation." Quarterly Journal of Economics 114 (1999), 739-767.

[Gibrat 30] R. Gibrat. "Une loi des réparations économiques: l'effet proportionnel." Bull. Statist. gén Fr. 19 (1930), 469.

[Gibrat 31] R. Gibrat. Les inegalites economiques. Paris: Libraire du Recueil Sirey, 1931.

[Gibson et al. 98] D. Gibson, J. Kleinberg, and P. Raghavan. "Inferring Web Communities from Link Topology." In Proceedings of the 9th ACM Conference on Hypertext and Hypermedia, pp. 225-234, New York: ACM Press, 1998.

[Gong et al. 01] W. Gong, Y.Liu, V. Misra, and D. Towsley. "On the Tails of Web Filesize Distributions." In Proceedings of the Thirty-Ninth Annual Allerton Conference on Communication, Control, and Computing, pp. 192-201. Urbana-Champaign, IL: Coordinated Science Laboratory, University of Illinois, 2001.

[Huberman and Adamic 99] B. A. Huberman and L. A. Adamic. "Evolutionary Dynamics of the World Wide Web." Technical Report, Xerox Palo Alto Research Center, 1999. Appears as a brief communication in Nature 401 (1999), 131.

[Huberman and Adamic 00] B. A. Huberman and L. A. Adamic. "The Nature of Markets in the World Wide Web." Quarterly Journal of Economic Commerce 1 (2000), $5-12$.

[Hull 97] J. C. Hull. Introduction to Futures and Options Markets, Third edition. Engelwood Cliffs, NJ: Prentice-Hall, Inc., 1997.

[Ito 51] K. Itô. Stochastic Differential Equations, Memoirs of the American Mathematical Society 4. Providence, RI: American Mathematical Society, 1951.

[Jain and Ramakumar 99] R. Jain and S. Ramakumar. "Stochastic Dynamics Modeling of the Protein Sequence Length Distribution in Genomes: Implications for Microbial Evolution." Physica A 273 (1999), 476-485.

[Kapteyn 1903] J. C. Kapteyn. Skew Frequency Curves in Biology and Statistics, Astronomical Laboratory. Groningen: Noordhoff, 1903.

[Kesten 73] H. Kesten. "Random Difference Equations and Renewal Theory for Products of Random Matrices." Acta Mathematica CXXXI (1973), 207-248. 
[Kleinberg et al. 99] J. Kleinberg, R. Kumar, P. Raghavan, S. Rajagopalan, and A. Tomkins. "The Web as a Graph: Measurements, Models, and Methods." In Proceedings of the International Conference on Combinatorics and Computing, Lecture Notes in Computer Science 1627, pp. 1-18. Berlin-Heidelberg: SpringerVerlag, 1999.

[Krapivsky and Redner 01] P. L. Krapivsky and S. Redner. "Organization of Growing Random Networks." Physical Review E 63 (2001), 066123-1-066123-14.

[Krugman 96] P. Krugman. The Self-Organizing Economy. Cambridge, MA: Blackwell, 1996.

[Kumar et al. 99a] R. Kumar, P. Raghavan, S. Rajagopalan, and A. Tomkins. "Extracting Large Scale Knowledge Bases from the Web." In Proceedings of the 25th VLDB Conference, pp. 639-650. San Francisco: Morgan Kaufmann, 1999.

[Kumar et al. 99b] R. Kumar, P. Raghavan, S. Rajagopalan, and A. Tomkins. "Trawling the Web for Emerging Cyber-Communities." In Proceedings of the 8th International World Wide Web Conference, pp. 403-415. Amsterdam: Elsevier Science, 1999.

[Kumar et al 00] R. Kumar, P. Raghavan, S. Rajagopalan, D. Sivakumar, A. Tomkins, and E. Upfal. "Stochastic Models for the Web Graph." In Proceedings of the 41st Annual Symposium on Foundations of Computer Science, pp. 57-65. New York: ACM Press, 2000.

[Kurtz 81] T. G. Kurtz, Approximation of Population Processes. Philadelphia, PA: SIAM, 1981.

[Leland et al. 94] W. E. Leland, M. S. Taqqu, W. Willinger, and D. V. Wilson. "On the Self-Similar Nature of Ethernet Traffic." IEEE/ACM Transactions on Networking (1994), 1-15.

[Li 96] W. Li. "Random Texts Exhibit Zipf's-Law-Like Word Frequency Distribution." IEEE Transactions on Information Theory 38:6 (1992), 1842-1845.

[Li 99] W. Li. "References on Zipf's Law." Available from World Wide Web (http://linkage.rockerfeller.edu/wli/zipf/), 1999.

[Mihail and Papadimitriou 02] M. Mihail and C. H. Papadimitriou. "On the Eigenvalue Power Law." In Proceedings of RANDOM 2002, pp. 254-262. BerlinHeidelberg: Springer-Verlag, 2002.

[Lotka 26] A. J. Lotka. "The Frequency Distribution of Scientific Productivity." Journal of the Washington Academy of Sciences 16 (1926), 317-323.

[Lu and Feng 98] J. Lu and Q. Feng. "Strong Consistency of the Number of the Vertices of Given Degrees in Nonuniform Random Recursive Trees." Yokohama Math. J. 45 (1998), 61-69.

[Mahmound et al. 93] H. Mahmound, R. Smythe, and J. Szymański. "On the Structure of Plane-Oriented Recursive Trees and their Branches. Random Structures and Algorithms 3 (1993), 255-266.

[Mandelbrot 53] B. Mandelbrot. "An Informational Theory of the Statistical Structure of Languages." In Communication Theory, edited by W. Jackson, pp. 486-502. Woburn, MA: Butterworth, 1953. 
[Mandelbrot 59] B. Mandelbrot. "A Note on a Class of Skew Distribution Function: Analysis and Critique of a Paper by H.A. Simon." Information and Control 2 (1959), 90-99.

[Mandelbrot 61a] B. Mandelbrot. "Final Note on a Class of Skew Distribution Functions: Analysis and Critique of a Model due to H.A. Simon." Information and Control 4 (1961), 198-216.

[Mandelbrot 61b] B. Mandelbrot. "Post Scriptum to 'Final Note'." Information and Control 4 (1961), 300-304.

[Mandelbrot 97] B. Mandelbrot. Fractals and Scaling in Finance. New York: SpringerVerlag, 1997.

[McAlister 1879] D. McAlister. "The Law of the Geometric Mean." Proceedings of the Royal Society 29 (1879), 367.

[Medina et al. 00] A. Medina, I. Matta, and J. Byers. "On the Origin of Power Laws in Internet Topologies." Computer Communication Review 30:2 (2000), 18-28.

[Miller 57] G. A Miller. "Some Effects of Intermittent Silence." American Journal of Psychology 70 (1957), 311-314.

[Mitzenmacher 02] M. Mitzenmacher. "Dynamic Models for File Size Distributions and Double Pareto Distributions." Preprint, available from World Wide Web (http://www.eecs.harvard.edu/ michaelm/NEWWORK/papers/), 2002.

[Mitzenmacher 01] M. Mitzenmacher. "A Brief History of Generative Models for Power Law and Lognormal Distributions. In Proceedings of the Thirty-Ninth Annual Allerton Conference on Communication, Control, and Computing, pp. 182-191, Urbana-Champaign, IL: Coordinated Science Laboratory, University of Illinois, 2001.

[Montroll and Schlesinger 82] E. W. Montroll and M. F. Shlesinger. "On $1 / f$ Noise and Other Distributions with Long Tails." Proceedings of the National Academy of Sciences 79 (1982), 3380-3383.

[Montroll and Schlesinger 83] E. W. Montroll and M. F. Shlesinger. "Maximum Entropy Formalism, Fractals, Scaling Phenomena, and $1 / f$ Noise: A Tale of Tails." Journal of Statistical Physics 32 (1983), 209-230.

[Nakajima and Higurashi 98] T. Nakajima and A. Higurashi. "A Use of Two-Channel Radiances for an Aeresol Characterization from Space." Geophysical Research Letters 25 (1998), 3815-3818.

[Pareto 1896] V. Pareto. Cours d'Economie Politique. Geneva: Droz, 1896.

[Paxson and Floyd 95] V. Paxson and S. Floyd. "Wide-Area Traffic: The Failure of Poisson Modeling." IEEE/ACM Transactions on Networking 3 (1995), 226-244.

[Perline 96] R. Perline. "Zipf's Law, the Central Limit Theorem, and the Random Division of the Unit Interval." Physical Review E 54:1 (1996), 220-223.

[Pittel 94] B. Pittel. "Note on the Height of Recursive Trees and $m$-ary Search Trees." Random Structures and Algorithms 5 (1994), 337-347.

[Reed 00] W. J. Reed. "The Pareto Law of Incomes-An Explanation and an Extension." Submitted. Available from World Wide Web (http://www.math.uvic.ca/faculty/reed/index.html), 2000. 
[Reed 01] W. J. Reed. "The Double Pareto-Lognormal Distribution-A New Parametric Model for Size Distribution." Available from World Wide Web (http://www.math.uvic.ca/faculty/reed/index.html), 2001.

[Reed and Hughes 02] W. J. Reed and B. D. Hughes. "From Gene Families and Genera to Incomes and Internet File Sizes: Why PowerLaws Are So Common in Nature." Available from World Wide Web (http://www.math.uvic.ca/faculty/reed/index.html), 2002.

[Simon 55] H. A. Simon. "On a Class of Skew Distribution Functions." Biometrika 42:3/4 (1955), 425-440.

[Simon 60] H. A. Simon. "Some Further Notes on a Class of Skew Distribution Functions." Information and Control 3 (1960), 80-88.

[Simon 61a] H. A. Simon. "Reply to 'Final Note'." Information and Control 4 (1961), $217-223$.

[Simon 61b] H. A. Simon. "Reply to Dr. Mandelbrot's Post Scriptum." Information and Control 4 (1961), 305-308.

[Smythe and Mahmound 95] R. Smythe and H. Mahmound. "A Survey of Recursive Trees." Theoretical Probability and Mathematical Statistics 51 (1995) 1-27.

[Sole et al. 00] R. V. Sole, D. Alonso, and A. McKane. "Connectivity and Scaling in S-species Model Ecosystems." Physica A 286 (2000), 337-344.

[Szymański 87] J. Szymański. "On a Nonuniform Random Recursive Tree." Annals of Discrete Mathematics 333 (1987), 297-306.

[Wheatland and Sturrock 96] M. S. Wheatland and P. A. Sturrock. "Avalanche Models of Solar Flares and the Distribution of Active Regions." The Astrophysical Journal 471 (1996), 1044-1048.

[Wormald 95] N. C. Wormald. "Differential Equations for Random Processes and Random Graphs." Annals of Appl. Prob. 5 (1995), 1217-1235.

[Yule 25] G. Yule. "A Mathematical Theory of Evolution Based on the Conclusions of Dr. J.C. Willis." F.R.S. Philosophical Transactions of the Royal Society of London (Series B) 213 (1925), 21-87.

[Zhu et al. 01] X. Zhu, J. Yu, and J. Doyle. "Heavy Tails, Generalized Coding, and Optimal Web Layout." In Proceedings of IEEE INFOCOM, pp. 1617-1626. Los Alamitos, CA: IEEE Press, 2001.

[Zipf 32] G. Zipf. Selective Studies and the Principle of Relative Frequency in Language. Cambridge, MA: Harvard University Press, 1932.

[Zipf 35] G. Zipf. The Psycho-Biology of Language: An Introduction to Dynamic Philology. Boston, MA; Houghton Mifflin Company, 1935.

[Zipf 39] G. Zipf. Human Behavior and the Principle of Least Effort. Cambridge, MA: Addison-Wesley, 1949. 
Michael Mitzenmacher, Harvard University, Division of Engineering and Applied Science, 33 Oxford St., Cambridge, MA 02138 (michaelm@eecs.harvard.edu)

Received November 27, 2002; accepted January 6, 2003. 